\title{
16 THE DESIGN AND IMPLEMENTATION OF A DATA LEVEL DATABASE INFERENCE DETECTION SYSTEM
}

\author{
Raymond W. Yip and Karl N. Levitt
}

\begin{abstract}
Inference is a way to subvert access control mechanisms of database systems. Most existing work on inference detection relies on analyzing functional dependencies in the database schema. This paper is an extension to our earlier effort in developing a data level inference detection system [13]. In this paper, we introduce the split query inference rule, make an extension to the overlapping inference rule, and provide an in depth discussion on the applications of the inference rules on union queries. Data level inference detection is inevitably expensive. We have developed a prototype of the inference detection system to evaluate its performance. The result shows that the system performs better with larger number of attributes and records in the database, and smaller number of projected attributes and return tuples of the queries. Therefore, the inference detection system could be practical when users retrieve a small amount of data compare to the size of the database.
\end{abstract}

\subsection{INTRODUCTION}

An inference occurs when a user infers data that the user is not allowed to access. In multilevel secure database systems, early work on inference detection employs a graph to represent the functional dependencies among attributes in the database schema. An inference occurs if there exists two paths between two attributes (or composite attributes), and the two paths are labeled at different 
classification levels $[5,1,10]$. The detected inference channel is eliminated by redesigning the database schema [8] or upgrading the paths that lead to the inference [11]. There is also work on incorporating external knowledge in detecting inference $[12,6,3]$. Detecting inference at the schema level is efficient as the detection is performed at the database design time. However, it has two drawbacks. First, the database schema does not capture all dependencies that occur in an instance of the database. Second, the existence of inference paths in the database schema does not necessary imply the users are making use of them to perform inference.

More recently, researchers look at the instance of the database to generate a richer set of functional dependencies for detecting inference. Hinke et al. use cardinality associations to discover potential inference channels [7]. Hale et al. incorporate imprecise and fuzzy database relations into their inference channel detection system [4]. Marks develops an inference detection system that prevents all possible inference by monitoring user queries with select clauses of the form " $A_{i}=a_{i}$ ", where $a_{i}$ is a constant [9]. Chang et al. use Bayesian estimation and network techniques to estimate missing data in the database $[2]$.

In this paper, we describe our effort in developing a data level inference detection system. We have identified six inference rules that users can use to infer data: split query, subsume, unique characteristic, overlapping, complementary, and functional dependence inference rules. Essentially, the six inference rules cover the set-subset, intersection, difference and union relationships among return tuples of queries. These rules are sound and they can be applied in any number of times, and in any order. The existence of these inference rules illustrates the inadequacy of the schema level inference detection approach.

However, data level inference detection is inevitably expensive, as it needs to keep track of all user queries and their return tuples. We have developed a prototype of the data level inference detection system to evaluate its performance. An earlier version of this paper is reported in [13]. In this paper, we introduce the split_query inference rule, make an extension to the overlapping inference rule, provide a detail description on the applications of the inference rules on union queries, and present a more complete experimental results. Because of lack of space, we omit the description of the unique characteristic and functional dependency inference rules. We also omit the use of examples to illustrate the inference rules. Interested readers can find them in [13].

This paper is organized as follows. In Section 2, we introduce the notations used in this paper. In Section 3, we present the inference rules. In Section 4, we discuss the applications of the inference rules on union queries. In Section 5 , we outline the inference detection algorithm. In Section 6 , we present our experimental results. In Section 7, we give a summary of the paper.

\subsection{NOTATIONS}

We consider a relational database that contains a single table. Multiple tables can be modeled as a universal relation as discussed in [9]. $t\left[A_{i}\right]$ denotes the 
attribute value of the tuple $t$ over the attribute $A_{i}$. A query is represented by a 2-tuple: (projected-attributes; selection-criterion), where projected-attributes is the set of attributes projected by the query, and selection-criterion is the logical expression that selects the return tuples of the query. No aggregation function (for example, maximum and average) is allowed to apply on the projectedattributes. Given a query $Q_{i},\left|Q_{i}\right|$ denotes the number of return tuples of $Q_{i}$, and $\left\{Q_{i}\right\}$ denotes the set of return tuples of $Q_{i}$. Unless otherwise stated, a set of return tuples is indeed a multiset of return tuples, that is, duplicated return tuples are retained. For each query $Q_{i}=\left\{A S_{i} ; S C_{i}\right\}, A S_{i}$ is expanded with $A_{i}$ when ' $A_{i}=a_{i}$ ' appears in $S C_{i}$ as a conjunct. An inferred query is a query that a user can infer its return tuples without directly issuing it to the database. A partial query $Q_{i}$ is a query that a user knows about $\left|Q_{i}\right|$ but not all the return tuples of $Q_{i}$. ' $\cap$ ', ' $U$ ', and ' $\backslash$ ' stand for the set intersection, union, and difference operations respectively.

A tuple $t$ projected over a set of attributes $S$ satisfies a logical expression $E$ if $E$ is evaluated to true when each occurrence of $A_{i}$ in $E$ is replaced with $t\left[A_{i}\right]$, for all $A_{i}$ in $S$. $t$ contradicts $E$ if $E$ is evaluated to false. A return tuple $t_{i}$ of a query $Q_{i}$ is indistinguishable from another return tuple $t_{j}$ of $Q_{j}$ if 1 ) $t_{i}[A]=t_{j}[A]$ for each attribute $\left.A \in\left(A S_{i} \cap A S_{j}\right), 2\right) t_{i}$ does not contradict $S C_{j}$, and 3$) t_{j}$ does not contradict $S C_{i}$. A tuple $t_{i}$ relates to another tuple $t_{j}$ if the two tuples are projected from the same tuple in the database. If $t_{i}$ relates to $t_{j}$, then $t_{i}$ is indistinguishable from $t_{j}$; but the reverse does not necessary hold. Given two queries, $Q_{1}$ and $Q_{2}$, we say that $Q_{1}$ is subsumed by $Q_{2}$, denoted as $Q_{1} \sqsubset Q_{2}$, if and only if 1) $S C_{1}$ logically implies $S C_{2}$ (denoted as $S C_{1} \Rightarrow S C_{2}$ ), or 2) for each return tuple $t_{1}$ of $Q_{1}, t_{1}$ satisfies $S C_{2}$. ' $\sqsubset$ ' is a reflexive, anti-symmetric, and transitive relation.

The goal of our inference detection system is to detect if a user can infer data using a series of queries. In particular, the system determines if a user can infer a return tuple of a query relates to a return tuple of another query. If so, the user can learn more about the return tuples.

\subsection{INFERENCE RULES}

In this section, we present four inference rules. Unless otherwise stated, all queries appear in the inference rules are not partial queries. We assume all the queries are issued by a single user, and there is no change to the database content. When two users are suspected of cooperating in performing inference, we run the inference detection system against their combined set of queries.

\subsubsection{Split Queries}

A query $Q_{i}$ can be split into two smaller queries when a user can identify the return tuples of $Q_{i}$ that relate to the return tuples of another query.

Inference Rule 1 (Split Queries) Given two queries $Q_{1}$ and $Q_{2}$. Express $S C_{2}$ in disjunctive normal form. If there exists a disjunct of $S C_{2}$ such that the set of attributes appear in the disjunct is a subset of $A S_{1}$, then generate two 
inferred queries: 1) $Q_{11}=\left(A S_{1} ; S C_{1} \wedge S C_{2}\right)$; and 2) $Q_{12}=\left(A S_{1} ; S C_{1} \wedge \neg S C_{2}\right)$. $Q_{2}$ may be a partial query. The return tuples of $Q_{11}$ are the return tuples of $Q_{1}$ that also satisfy $S C_{2}$. The return tuples of $Q_{12}$ are the return tuples of $Q_{1}$ that does not satisfy $S C_{2}$.

When $Q_{1}$ projects all attributes that appear in a disjunct of $S C_{2}$, a user can identify the return tuples of $Q_{1}$ that satisfy $S C_{2}$. Hence, the user can divide the return tuples of $Q_{1}$ into two sets: those that satisfy both $S C_{1}$ and $S C_{2}$, and those that satisfy $S C_{1}$ but not $S C_{2}$.

\subsubsection{Subsume Inference}

In this section, we describe inference making use of the subsume relations among queries.

Inference Rule 2 (Subsume) Given two queries $Q_{1}$ and $Q_{2}$, such that $Q_{1} \sqsubset$ $Q_{2}$.

SI1 If there is an attribute $A$ in $\left(A S_{2} \backslash A S_{1}\right)$, such that all return tuples of $Q_{2}$ take the same attribute value $a$ over $A$, then for each return tuple $t_{1}$ of $Q_{1}, t_{1}[A]=a . Q_{1}$ may be a partial query.

SI2 If a return tuple $t_{1}$ of $Q_{1}$ is indistinguishable from exactly one return tuple $t_{2}$ of $Q_{2}$, then $t_{1}$ relates to $t_{2} . Q_{1}$ may be a partial query.

SI3 Let $S$ be the set of return tuples of $Q_{2}$ that are distinguishable from the return tuples of $Q_{1}$. If $|S|=\left(\left|Q_{2}\right|-\left|Q_{1}\right|\right)$, generate two inferred queries from $Q_{2}$ : 1) $Q_{21}=\left(A S_{2} ; S C_{2} \wedge \neg S C_{1}\right)$ with $S$ as the set of return tuples; and 2) $Q_{22}=\left(A S_{2} ; S C_{2} \wedge S C_{1}\right)$ with $\left(\left\{Q_{2}\right\} \backslash S\right)$ as the set of return tuples. If $|S|<\left(\left|Q_{2}\right|-\left|Q_{1}\right|\right)$, generate an inferred partial query: $Q_{23}=\left(A S_{2} ; S C_{2} \wedge \neg S C_{1}\right)$ with $S$ as the partial set of return tuples, and $\left|Q_{23}\right|=\left(\left|Q_{2}\right|-\left|Q_{1}\right|\right)$.

$Q_{1} \sqsubset Q_{2}$ implies that for each return tuple $t_{1}$ of $Q_{1}$, there is a return tuple $t_{2}$ of $Q_{2}$ such that $t_{1}$ relates to $t_{2}$. SI1 says that when all return tuples of $Q_{2}$ share a common attribute value, say $a$, over an attribute $A$, a user can infer that each return tuple of $Q_{1}$ also takes the attribute value $a$ over the attribute $A$. This is because for each return tuple $t_{1}$ of $Q_{1}$, no matter which return tuple $t_{2}$ of $Q_{2}$ that relates to $t_{1}, t_{2}[A]=a$. Hence, $t_{1}[A]$ must be equal to $a$.

SI2 says that if $t_{1}$ of $Q_{1}$ is indistinguishable from exactly one return tuple $t_{2}$ of $Q_{2}$, then $t_{1}$ relates to $t_{2}$. This is because $Q_{1} \sqsubset Q_{2}$ implies that there is at least one return tuple of $Q_{2}$ that is indistinguishable from each return tuple of $Q_{1}$. Now, if $t_{1}$ of $Q_{1}$ is indistinguishable from one and only one return tuple $t_{2}$ of $Q_{2}$, then we can conclude that $t_{1}$ relates to $t_{2}$.

$S I 3$ says that if a user identifies all the return tuples of $Q_{2}$ that relate to the return tuples of $Q_{1}$, then the user can infer these two queries from $Q_{2}:\left(A S_{2}\right.$; $S C_{1} \wedge S C_{2}$ ) which includes return tuples of $Q_{2}$ that relate to the return tuples of $Q_{1}$, and $\left(A S_{2} ; S C_{2} \wedge \neg S C_{1}\right)$ which includes return tuples of $Q_{2}$ that do not relate to the return tuples of $Q_{1}$. 


\subsubsection{Overlapping Inference}

In this section, we describe the overlapping inference rule.

\section{Inference Rule 3 (Overlapping)}

OI1 Given $Q_{1} \sqsubset Q_{2}$, and $Q_{1} \sqsubset Q_{3}$. Let $S_{2}$ be the set of return tuples of $Q_{2}$ that are indistinguishable from the return tuples of $Q_{3}$. If $\left|S_{2}\right|=\left|Q_{1}\right|$, and a return tuple $t_{2}$ of $Q_{2}$ is indistinguishable from exactly one return tuple $t_{3}$ of $Q_{3}$, then $t_{2}$ relates to $t_{3}$. Similarly, let $S_{3}$ be the set of return tuples of $Q_{3}$ that are indistinguishable from the return tuples of $Q_{2}$. If $\left|S_{3}\right|=\left|Q_{1}\right|$, and a return tuple $t_{3}$ of $Q_{3}$ is indistinguishable from exactly one return tuple $t_{2}$ of $Q_{2}$, then $t_{3}$ relates to $t_{2}$. Suppose $\left|Q_{1}\right|=\left|S_{2}\right|=\left|S_{3}\right|$. If a return tuple $t_{1}$ of $Q_{1}$ is indistinguishable from exactly one return tuple $t_{2}$ in $S_{2}$, then $t_{1}$ relates to $t_{2}$. Also, if $t_{1}$ is indistinguishable from exactly one return tuple $t_{3}$ in $S_{3}$, then $t_{1}$ relates to $t_{3}$. $Q_{1}$ may be a partial query.

OI2 Given a query $Q_{1}$, and a set of queries, $Q S=\left\{Q_{2}, \ldots, Q_{n}\right\}$, where $n \geq 3$. Suppose $S C_{1} \Leftrightarrow\left(S C_{2} \vee \ldots \vee S C_{n}\right)$, and for each $Q_{i}$ in $Q S, Q_{i} \sqsubset Q_{1}$. If the number of distinguishable tuples in $Q S=\left|Q_{1}\right|$, then any pair of indistinguishable tuples relate to each other.

OI3 When $O I 1$ is applied and all the related return tuples between $Q_{2}$ and $Q_{3}$ have been identified, generate the following two inferred queries from $Q_{2}$ : 1) $Q_{21}=\left(A S_{2} ; S C_{2} \wedge \neg S C_{3} \wedge \neg S C_{1}\right)$ with $\left\{Q_{2}\right\} \backslash S_{2}$ as the set of return tuples; and 2) $Q_{22}=\left(A S_{2} ; S C_{2} \wedge S C_{3}\right)$ with $S_{2}$ as the set of return tuples. Similarly generate two inferred queries from $Q_{3}$. When OI2 is applied, generate possibly four inferred queries for each pair of queries that have overlapping return tuples.

Given that $Q_{1} \sqsubset Q_{2}$ and $Q_{1} \sqsubset Q_{3}$, the number of return tuples of $Q_{2}$ that relate to return tuples of $Q_{3}$ must be at least $\left|Q_{1}\right|$. OI1 identifies the cases where a user can infer the related return tuples among the three queries. When $Q_{1}$ implies three or more queries, $O I 1$ is applied to two of them at a time.

We illustrate OI2 using three queries, $Q_{1}, Q_{2}$, and $Q_{3}$, where $Q_{1} \sqsubset Q_{3}$, $Q_{2} \sqsubset Q_{3}$, and $S C_{3} \Leftrightarrow S C_{1} \vee S C_{2}$. Let $N$ be the number of indistinguishable tuples in $Q_{1}$ and $Q_{2}$. As $S C_{3} \Leftrightarrow S C_{1} \vee S C_{2}$, each return tuple of $Q_{3}$ relates to a return tuple in $Q_{1}$ or $Q_{2}$. Hence, $N \geq\left|Q_{3}\right|$. Furthermore, as $Q_{1} \sqsubset Q_{3}$ and $Q_{2} \sqsubset Q_{3}$, each distinguishable tuple in $Q_{1}$ and $Q_{2}$ relates to a return tuple of $Q_{3}$. Hence, $N \leq\left|Q_{3}\right|$. Therefore, $N=\left|Q_{3}\right|$. When a user find out that the number of indistinguishable tuples in $Q_{1}$ and $Q_{2}$ equals $\left|Q_{3}\right|$, the user can infer that for each return tuple $t_{1}$ of $Q_{1}$ that is indistinguishable from a return tuple $t_{2}$ of $Q_{2}, t_{1}$ relates to $t_{2}$.

\subsubsection{Complementary Inference}

The complementary inference rule performs inference by eliminating tuples that are not related to one another. 
Inference Rule 4 (Complementary Inference) Given four queries, $Q_{1}, Q_{2}$, $Q_{3}$, and $Q_{4}$, where $Q_{1} \sqsubset Q_{2}$, and $Q_{3} \sqsubset Q_{4}$. Also, the return tuples of $Q_{1}$ that relate to the return tuples of $Q_{3}$ are identified (for example using the overlapping inference rule), and the return tuples of $Q_{2}$ that relate to the return tuples of $Q_{4}$ are identified. If one of the following three conditions holds,

1. for each return tuple $t_{1}$ of $Q_{1}$ that does not relate to any return tuple of $Q_{3}, t_{1}$ is distinguishable from all return tuples of $Q_{4}$,

2. $Q_{4} \sqsubset Q_{3}$, or

3. $\left|Q_{3}\right|=\left|Q_{4}\right|$,

then $Q_{1}^{\prime} \sqsubset Q_{2}^{\prime}$, where $Q_{1}^{\prime}=\left(A S_{1} ; S C_{1} \wedge \neg S C_{3}\right)$, and $Q_{2}^{\prime}=\left(A S_{2} ; S C_{2} \wedge \neg\right.$ $\left.S C_{4}\right) .\left\{Q_{1}^{\prime}\right\}$ is the set of return tuples of $Q_{1}$ that do not relate to any return tuple of $Q_{3}$, and $\left\{Q_{2}^{\prime}\right\}$ is the set of return tuples of $Q_{2}$ that do not relate to any return tuple of $Q_{4}$.

As $Q_{1} \sqsubset Q_{2}$ and $\left\{Q_{1}^{\prime}\right\} \subset\left\{Q_{1}\right\}$, each return tuple of $Q_{1}^{\prime}$ relates to a return tuple of $Q_{2}$. Condition (1) says that each return tuple of $Q_{1}^{\prime}$ does not relate to any return tuple of $Q_{4}$. Hence, each return tuple of $Q_{1}^{\prime}$ relates to a return tuple of $Q_{2}^{\prime}$. Condition (2) or (3) implies $\left(\left(Q_{3} \sqsubset Q_{4}\right) \wedge\left(Q_{4} \sqsubset Q_{3}\right)\right)$. By removing from $Q_{1}$ and $Q_{2}$ the "same" set of return tuples, we have $Q_{1}^{\prime} \sqsubset Q_{2}^{\prime}$.

It should be noted that in some cases, the inference as obtained from the complementary inference rule can also be obtained from the overlapping inference rule. For example, consider four queries $Q_{1}, Q_{2}, Q_{3}$, and $Q_{4}$, where $Q_{1} \sqsubset Q_{2}$, and $Q_{3} \sqsubset Q_{4}$. Suppose the overlapping inference rule can be applied to identify the related tuples between $Q_{1}$ and $Q_{3}$, and between $Q_{2}$ and $Q_{4}$. These result in the generation of two inferred queries: 1) $Q_{1}^{\prime}=\left(A S_{1} ; S C_{1} \wedge \neg S C_{3}\right)$; and 2) $Q_{2}^{\prime}=\left(A S_{2} ; S C_{2} \wedge \neg S C_{4}\right)$. If $\left(S C_{1} \wedge \neg S C_{3}\right) \Rightarrow\left(S C_{2} \wedge \neg S C_{4}\right)$, then we have $Q_{1}^{\prime} \sqsubset Q_{2}^{\prime}$ which is the same result as obtained by applying the complementary inference rule to the four queries. However, $S C_{1} \Rightarrow S C_{2}$ and $S C_{3} \Rightarrow S C_{4}$ does not necessary implies $\left(S C_{1} \wedge \neg S C_{3}\right) \Rightarrow\left(S C_{2} \wedge \neg S C_{4}\right)$. When this implication does not hold, the complementary inference rule is needed to perform the inference.

\subsection{INFERENCE WITH UNION QUERIES}

The inference rules can be applied to unions of queries. We call a union of queries a 'union query'. In contrast, a user query or an inferred query is called a 'simple query'. If $Q_{u}$ is a union query consists $Q_{i}, \ldots$, and $Q_{j}$, then $A S_{u}=$ $\left(A S_{i} \cap \ldots \cap A S_{j}\right)$, and $S C_{u}=\left(S C_{i} \vee \ldots \vee S C_{j}\right)$. Note that $A S_{u}$ might be equal to $\emptyset$. The applications of the split query, unique characteristic and functional dependency inference rules on union queries are similar to their applications on simple queries. Hereafter, we only discuss the applications of the subsume, overlapping, and complementary inference rules on union queries. 


\subsubsection{Subsume Inference Rule on Union Queries}

Consider the applications of the subsume inference rule on union queries when the union queries are subsumed by other queries. Let $Q_{u}=\left\{Q_{i}, \ldots, Q_{j}\right\}$ be a union query, and $Q_{u} \sqsubset Q_{1}$. We show that inference obtained by applying the subsume inference rule on $\left(Q_{i} \cup \ldots \cup Q_{j}\right) \sqsubset Q_{1}$ can also be obtained by applying the subsume inference rule on $Q_{i} \sqsubset Q_{1}, \ldots$, and $Q_{j} \sqsubset Q_{1}$.

Consider the applications of $S I 1$. If there is an attribute $A$ in $\left(A S_{1} \backslash A S_{u}\right)$, such that all return tuples of $Q_{1}$ take the same attribute value $a$ over $A$, then for each return tuple $t_{u}$ of $Q_{u}, t_{u}[A]=a$. This implies that for each return tuple $t$ of a simple query of $Q_{u}, t[A]=a$. This is the same as if the $S I 1$ is applied to $Q_{i}$ and $Q_{1}$, where $Q_{i} \sqsubset Q_{1}$, for each simple query $Q_{i}$ of $Q_{u}$.

Consider the applications of SI2. If there exists a tuple $t_{u}$ in $Q_{u}$ that is indistinguishable from exactly one return tuple $t_{1}$ of $Q_{1}$, there exists at least one simple query $Q_{i}$ of $Q_{u}$ such that $t_{u}$ relates to a return tuple $t_{i}$ of $Q_{i}$. Now, $t_{i}$ is indistinguishable from $t_{1}$ of $Q_{1}$. Hence, when SI2 is applicable to infer that $t_{u}$ of $Q_{u}$ relates to $t_{1}$ of $Q_{1}$, it is also applicable to infer that $t_{i}$ of $Q_{i}$ relates to $t_{1}$ of $Q_{1}$.

Consider the applications of SI3. When all the related tuples between $Q_{u}$ and $Q_{1}$ are identified, two inferred queries are generated from $\left.Q_{1}: 1\right) Q_{u 1}=$ $\left(A S_{1} ; S C_{1} \wedge \neg S C_{u}\right)$; and 2) $Q_{u 2}=\left(A S_{1} ; S C_{1} \wedge S C_{u}\right)$. We show that these two queries can also be generated from the simple queries of $Q_{u}$ and $Q_{1}$. Note that when all the related tuples between $Q_{u}$ and $Q_{1}$ have been identified, all related tuples among the simple queries of $Q_{u}$ are also identified. Without loss of generality, suppose $Q_{u}=\left\{Q_{2}, Q_{3}\right\}$. The application of $S I 3$ on $Q_{1}$ and $Q_{2}$ generates two inferred queries: 1) $Q_{21}=\left(A S_{1} ; S C_{1} \wedge \neg S C_{2}\right)$; and 2) $Q_{22}=$ $\left(A S_{1} ; S C_{1} \wedge S C_{2}\right)$. Similarly, the application of $S I 3$ on $Q_{1}$ and $Q_{3}$ generates two inferred queries: 1) $Q_{31}=\left(A S_{1} ; S C_{1} \wedge \neg S C_{3}\right)$; and 2) $Q_{32}=\left(A S_{1} ; S C_{1} \wedge\right.$ $\left.S C_{3}\right)$. Now, $Q_{21}$ and $Q_{31}$ are both generated from $Q_{1}$, and we can generate the following inferred query for their related tuples: $\left(A S_{1} ; S C_{1} \wedge \neg S C_{2} \wedge \neg S C_{3}\right)$ which equals $Q_{u 1}$. $Q_{22}$ and $Q_{32}$ are both generated from $Q_{1}$, and we can identify the related tuple between them. The union of these two queries is $\left(A S_{1} ; S C_{1} \wedge\left(S C_{2} \vee S C_{3}\right)\right)$ which equals $Q_{u 2}$. Therefore, we do not need to consider the applications of the subsume inference rule when the union query is subsumed by other queries.

Consider the case where union queries subsume other queries, say $Q_{1} \sqsubset Q_{u}$. $S I 1$ is applied as follows. If for each return tuple $t$ of any simple query of $Q_{u}$, $t[A]=a$, then $t_{1}[A]=a$ for each return tuple $t_{1}$ of $Q_{1}$. SI2 is applied as follows. If there is a return tuple $t_{1}$ of $Q_{1}$ that is indistinguishable from a set of return tuples $S$ from the simple queries of $Q_{u}$, where all tuples in $S$ relate to one another, then $t_{1}$ relates to each tuple in $S$. SI3 is applied similarly. Note that the subsume inference rule can still be applied when the simple queries of $Q_{u}$ have no common projected attribute. 


\subsubsection{Overlapping and Complementary Inference Rule on Union Queries}

Consider the applications of $O I 1$. Given three queries, $Q_{1}, Q_{2}$, and $Q_{u}$, where $Q_{u}$ is a union query. Suppose $Q_{u} \sqsubset Q_{1}$ and $Q_{u} \sqsubset Q_{2}$. If $O I 1$ is to be applied to identify the related return tuples among $Q_{2}$ and $Q_{3},\left|Q_{u}\right|$ must be known. That is, the number of related tuples, if any, between the simple queries are identified. Now, suppose $Q_{1} \sqsubset Q_{u}$ and $Q_{1} \sqsubset Q_{2}$. If $O I 1$ is to be applied to identify the related return tuples between $Q_{u}$ and $Q_{2}$, then the user must has already identified those related tuples among the simple queries in $Q_{u}$. Also, the user has to identify the return tuples of $Q_{u}$ that are indistinguishable from the return tuples of $Q_{2}$, and the number of these return tuples equals $\left|Q_{1}\right|$.

Consider the applications of OI2. Suppose there is a set of queries $Q S=$ $\left\{Q_{2}, \ldots, Q_{n}, Q_{u}\right\}$ such that for each query $Q_{i} \in Q S, Q_{i} \sqsubset Q_{1}$. OI2 is applicable when the related tuples among the queries in $Q S$ are identified. That is, the related return tuples, if any, between $Q_{u}$ and other queries in $Q S$ have to be identified. $O I 3$ is applied similar to the case with simple queries.

Note that the overlapping inference rule can still be applied when $A S_{u}=$ $\emptyset$. For example, let $Q_{u}=\left\{Q_{u 1}, Q_{u 2}\right\}$. If $S C_{u 1} \wedge S C_{u 2}=$ false, the user can conclude that there is no related return tuple between $Q_{u 1}$ and $Q_{u 2}$, and $\left|Q_{u}\right|=\left|Q_{u 1}\right|+\left|Q_{u 2}\right|$.

Consider the applications of the complementary inference rule on the union queries. Suppose there are four queries $Q_{1}, Q_{2}, Q_{3}$, and $Q_{u}$, where $Q_{u}$ is a union query, $Q_{1} \sqsubset Q_{2}$, and $Q_{3} \sqsubset Q_{u}$. To apply the complementary inference rule on these four queries, the related return tuples among the simple queries in $Q_{u}$ that aiso relate to return tuples of $Q_{2}$ must have been identified. Similarly for the case when $Q_{1}, Q_{2}$, or $Q_{3}$ is a union query.

\subsection{INFERENCE DETECTION ALGORITHMS}

In this section, we outline the inference detection algorithms. Figure 16.1 shows the main function INFERENCE $\left(U, Q_{i}\right)$, which is called each time a user $U$ issues a query $Q_{i}$ to the database. The function maintains two sets: GEN and $E X P$. GEN is initialized with the user issued query $Q_{i}$, and is subsequently being added with inferred queries generated by the inference rules. Each query in $G E N$ is compared with previously issued or inferred queries for user $U$ (denoted as PREV_QUERY $(U))$ to determine if the inference rules are applicable to them. EXP is the set of tuples that are expanded during the applications of the inference rules. The results of the applications of inference rules are generations of inferred queries and expansions of some return tuples of queries. Given a tuple $t_{1}$ projected over a set of attributes $A S_{1}$, and another tuple $t_{2}$ projected over a set of attributes $A S_{2}$. If $t_{1}$ and $t_{2}$ are found to be related to each other, $t_{1}$ is expanded as follows: for each attribute $A \in A S_{2} \backslash A S_{1}, t_{1}[A]=t_{2}[A] . t_{2}$ is expanded similarly.

After a tuple is expanded, the query that returns the expanded tuple might be eligible in further applications of inference rules. Hence, the function checks if the inference rules are applicable to the query. INFERENCE is a terminating 
function, as the number of inferences is bound by the size of the database. In each call to the INFERENCE function, all queries in GEN are processed before the expanded tuples in EXP. This avoids repeatedly processing the same tuple which is expanded more than once after queries in GEN are processed.

INFERENCE $\left(U, Q_{i}\right)$ :

1. $\quad$ initialize $G E N$ with $Q_{i}$;

2. $E X P \leftarrow \emptyset$;

3. $\quad G E N_{-} Q \leftarrow \emptyset$;

4. $\quad E X P \_Q \leftarrow \emptyset$;

5. $\quad$ while $(G E N \neq \emptyset$ or $E X P \neq \emptyset)$ do

6. $\quad$ if $G E N \neq \emptyset$ then

7. $\quad Q_{j} \leftarrow$ a query in $G E N$;

8. remove $Q_{j}$ from $G E N$

9. $\quad G E N_{-} Q \leftarrow G E N_{-} Q \cup\left\{Q_{j}\right\}$;

10. else if $E X P \neq \emptyset$ then

11. $Q_{j} \leftarrow$ a query that returns a tuple in $E X P$;

12. $E X P_{-} Q \leftarrow E X P_{-} Q \cup\left\{Q_{j}\right\}$;

13. $t s \leftarrow$ return tuples of $Q_{j}$ in $E X P$;

14. remove return tuples of $Q_{j}$ from $E X P$;

15. for each $Q_{k} \in P R E V_{-} Q U E R Y(U)$ do

16. $\quad E X P \leftarrow \operatorname{UNIQUE}\left(Q_{j}, Q_{k}, t s, E X P\right)$;

17. $\quad G E N \leftarrow \operatorname{SPLIT}$ QQUERY $\left(Q_{j}, Q_{k}, G E N\right)$;

18. if $Q_{j} \sqsubset Q_{k}$ then

19.

20.

21.

22.

23.

24.

25.

26. FIND_UNION $\left(U, G E N_{-} Q, E X P_{-} Q\right)$;

$(G E N, E X P) \leftarrow \operatorname{SUBSUME}\left(Q_{j}, Q_{k}, G E N, E X P\right)$;

$(G E N, E X P) \leftarrow$ OVERLAP $\left(U, Q_{j}, Q_{k}, G E N, E X P\right)$;

$G E N \leftarrow \operatorname{COMPLEMENTARY}\left(Q_{j}, Q_{k}, G E N\right)$;

else if $Q_{k} \sqsubset Q_{j}$ then

$(G E N, E X P) \leftarrow \operatorname{SUBSUME}\left(Q_{k}, Q_{j}, G E N, E X P\right) ;$

$(G E N, E X P) \leftarrow$ OVERLAP $\left(U, Q_{k}, Q_{j}, G E N, E X P\right)$;

$G E N \leftarrow$ COMPLEMENTARY $\left(Q_{k}, Q_{j}, G E N\right)$;

Figure 16.1 The inference function.

The function UNIQUE has three input parameters: $Q_{j}, Q_{k}$, and $t s$. The function checks if unique characteristic can be determined between the two queries $Q_{j}$ and $Q_{k}$. For each expanded return tuple in $t s$, the function checks if the expanded return tuple and another return tuple have common unique characteristics. If so, the two return tuples are expanded with each other. The functions SPLIT_QUERY, SUBSUME, OVERLAP, and COMPLEMENTARY operate as described in the corresponding inference rules, and we omit the presentations of their algorithms. The FIND_UNION function checks if there are unions of query that satisfy the subsume relations with other queries. If so, the inference rules are applied to them. 


\subsection{EXPERIMENTAL RESULTS}

We have developed a prototype of the inference detection system in about 4,000 lines of Perl code. We have implemented the split query, subsume, unique characteristic, overlapping (except OI2), and complementary inference rules. The system also handles applications of the inference rules on union queries. We run our experiments with randomly generated tables and user queries. Each table has $N_{a t t r}$ number of attributes, and $N_{\text {rec_num }}$ number of records. The primary key of the table is a single attribute. All attributes are of integer types. Attribute values in the table are uniformly distributed between 0 and $\left(N_{\text {data_dist }} \times N_{\text {rec_num }}\right)$, where $0<N_{\text {data_dist }} \leq 1$. We also randomly generate $N_{\text {query_num }}$ number of user queries. Each query projects $N_{\text {proj }}$ number of attributes from the table. The selection criterion of each query is a conjunction of $N_{\text {cond }}$ number of conjuncts. Each conjunct is of the form ' $A_{i} o p a_{i}$ ', where $A_{i}$ is an attribute from the table, $o p$ is one of the comparison operators $(>, \geq$, $\leq,<$, and $=$ ), and $a_{i}$ is an attribute value. Each query has $N_{\text {ret_tuple }}$ number of return tuples. We approximate the evaluation of a logical implication $C_{i} \Rightarrow C_{j}$ by checking if the tuples selected by $C_{i}$ is also selected by $C_{j}$, and that the set of attributes appear in $C_{j}$ is a subset of those appear in $C_{i}$. We collect the following two data to measure the system performance: 1) average number of seconds used to process one query. 2) number of times the inference rules are applied.

We ran six experiments to determine how the characteristics of the database and the queries affect the system performance. For the database, we consider the following characteristics: 1) the number of tuples in the database; 2) the number of attributes in the database; and 3) the amount of duplication of the data values. For the queries, we consider the following characteristics: 1) the number of attributes projected by the queries; 2) the number of conjuncts in the selection criteria; 3) the number of queries being issued; and 4) the number of tuples returned by the queries. The experimental results of running the inference detection system on a Sun SPARC 20 workstation are shown in Figure 16.2-Figure 16.7.

Experiment 1 investigates the effect of the number of attributes and the amount of data duplication in the database on the system performance. In this experiment, we choose the following parameter values: $N_{\text {rec_num }}=1000$, $N_{\text {ret_tuple }}=50, N_{\text {proj }}=4, N_{\text {cond }}=3$, and $N_{\text {query_num }}=500 . N_{\text {attr }}$ is varied with the following values: $40,60,80,100,120$, and $140 . N_{\text {data_dist }}$ is varied with the following values $25 \%, 50 \%, 75 \%$, and $100 \%$. Figure 16.2 shows the results in a graph plotted with the average query processing time (in seconds) against the number of attributes in the database. Consider each individual line in Figure 16.2. It shows that the system runs faster as $N_{\text {attr }}$ increases from 40 to 140 . With a fixed type of queries, the larger the number of attributes in the table, the lesser the amount of overlapping among the return tuples of queries. This results in lesser subsume relations hold among queries, and hence the smaller the number of inferences. 
Consider the four lines in Figure 16.2. They correspond to the cases where $N_{\text {data_dist }}=25 \%, 50 \%, 75 \%$, and $100 \%$. The lower the value of $N_{\text {data_dist }}$, the more duplication of the data in the database. Intuitively, the higher the duplication of the data, the lesser the number of distinguishable return tuples, and hence the smaller number of inferences. This is ture in some cases. However, in general the results do not show a significant effect of data duplication on the system performance.

Experiment 2 investigates the effect of the number of return tuples of queries on the system performance. Figure 16.3 shows the results for $N_{\text {rec_num }}=1000$, $N_{\text {data_dist }}=50 \%, N_{\text {proj }}=4, N_{\text {cond }}=3$, and $N_{\text {query_num }}=500 . N_{\text {ret_tuple }}$ takes the values of $50,100,150,200$, and 250 , and $N_{\text {attr }}$ takes the values of 80 and 120. The figure shows that the system runs slower as $N_{\text {ret_tuple }}$ increases. The larger the number of return tuples, the longer it takes for the system to process them. Also, the more the number of tuples returned by the queries, the more the number of occurrences of inferences, and also the more the number of inferred queries being generated.

Experiment 3 investigates the effect of the number of projected attributes in queries on the system performance. Figure 16.4 shows the results for $N_{\text {rec_num }}$ $=1000, N_{\text {query_num }}=500, N_{\text {data_dist }}=50 \%, N_{\text {attr }}=80$, and $N_{\text {ret_tuple }}=50$. $N_{\text {proj }}$ takes the values of $4,5,6,7$, and $8 . N_{\text {cond }}$ takes the values of $4,5,6$, and 7. It shows that the system runs slower as $N_{\text {proj }}$ increases. This is because the more the number of attributes projected by the queries, the more overlapping among the return tuples of queries, and hence the more number of inferences.

Experiment 4 investigates the effect of the number of conjunts in the selection criteria on the system performance. Figure 16.5 shows the results for $N_{\text {rec_num }}$ $=1000, N_{\text {query_num }}=500, N_{\text {data_dist }}=50 \%, N_{\text {attr }}=80$, and $N_{\text {ret_tuple }}=50$. $N_{\text {cond }}$ takes the values of $3,4,5,6$, and 7 . $N_{\text {proj }}$ takes the values of $4,5,6$, and 7 . It shows that the system runs faster as $N_{\text {cond }}$ increases. This is because the larger the number of conjuncts in the selection criteria of the queries, the lesser the chance that the subsume relations hold among the queries, and hence the smaller number of occurrences of inferences. However, the effect is not significant when $N_{\text {cond }}>3$.

Experiment 5 investigates the effect of the number of tuples in the database on the system performance. Figure 16.6 shows the result for $N_{\text {data_dist }}=50 \%$, $N_{\text {attr }}=80, N_{\text {ret_tuple }}=50, N_{\text {query_num }}=500, N_{\text {proj }}=4$, and $N_{\text {cond }}=3$. $N_{\text {rec_num }}$ is varied with the following values: $1000,2500,5000,7500$, and 10000 . It shows that the system runs faster as the number of tuples of the database increases. As the size of the database increases, the possible amount of overlapping among the queries decreases, and hence the lesser number of inferences. For $N_{\text {ret_tuple }}=10000$, the set of queries happen to generate more inferences than the case for $N_{\text {ret_tuple }}=5000$ or 7500 , and hence it has a longer running time.

Experiment 6 investigates the effect of the number of queries on the system performance. Figure 16.7 shows the results for $N_{\text {rec_num }}=1000, N_{\text {data_dist }}=$ $50 \%, N_{\text {attr }}=80, N_{\text {ret_tuple }}=30, N_{\text {proj }}=4$, and $N_{\text {cond }}=3 . N_{\text {rec_number }}$ takes 
the values of $200,400,600,800,1000$, and 1200 . It shows that the system runs slower as the number of queries to be processed increases. This is because the more the number of queries, the more the number of inferences. Also, as each user query needs to be compared with previously issued queries for the subsume relations, the more the number of queries, the longer it takes to determine all possible subsume relations.

\subsection{SUMMARY}

In this paper, we describe our effort in developing a data level inference detection system. We have identified six inference rules: split query, subsume, unique characteristic, overlapping, complementary, and functional dependency inference rules. We have also discussed the applications of the inference rules on union queries. The existence of these inference rules shows that simply using functional dependencies to detect inference is inadequate. We have developed a prototype of the inference detection system using Perl on a Sun SPARC 20 workstation.

Although the data level inference detection approach is inevitably expensive, there are cases where the uses of such approach is practical. As shown in our experimental results, the system generally performs better with a larger size of the database, and queries that return smaller number of tuples and project smaller number of attributes. The system running time becomes high when queries retrieve a large amount of data from the database, and there are large amount of overlapping among query results. However, when a user issues such type of queries, it is suspicious that the user is attempting to infer associations among the data.

\section{Acknowledgments}

The research reported in this paper is supported by the National Security Agency University Research Program under Contract DOD MDA904-96-1-0117, and by the CIA Office of Research and Development under Contract 96F 154000-000.

\section{References}

[1] Leonard J. Binns. Inference through secondary path analysis. In Proc. 6th IFIP WG11.3 Workshop on Database Security, pages 195-209, August 1992.

[2] LiWu Chang and Ira S. Moskowitz. Bayesian methods applied to the database inference problem. In Proc. 12th IFIP WG11.3 Workshop on Database Security, July 1998.

[3] Harry S. Delugach and Thomas H. Hinke. Wizard: A database inference analysis and detection system. IEEE Transactions on Knowledge and Data Engineering, 8(1):56-66, 1996.

[4] John Hale and Sujeet Shenoi. Catalytic inference analysis: Detection inference threats due to knowledge discovery. In Proceedings of the 1997 
IEEE Symposium on Research in Security and Privacy, pages 188-199, May 1997.

[5] Thomas H. Hinke. Inference aggregation detection in database management systems. In Proceedings of the 1988 IEEE Symposium on Security and Privacy, pages 96-106, April 1988.

[6] Thomas H. Hinke, Harry S. Delugach, and Asha Chandrasekhar. Layered knowledge chunks for database inference. In Proc. 7th IFIP WG11.3 Workshop on Database Security, pages 275-295, September 1993.

[7] Thomas H. Hinke, Harry S. Delugach, and Randall Wolf. A framework for inference-directed data mining. In Proc. 10th IFIP WG11.3 Workshop on Database Security, pages 229-239, July 1996.

[8] Teresa F. Lunt. Aggregation and inference: Facts and fallacies. In Proceedings of the 1989 IEEE Symposium on Security and Privacy, pages 102-109, May 1989.

[9] Donald G. Marks. Inference in mls database systems. IEEE Transactions on Knowledge and Data Engineering, 8(1):46-55, February 1996.

[10] Xiaolei Qian, Mark E. Stickel, Peter D. Karp, Teresa F. Lunt, and Thmoas D. Garvey. Detection and elimination of inference channels in multilevel relational database systems. In Proceedings of the 1993 IEEE Symposium on Research in Security and Privacy, pages 196-205, May 1993.

[11] Mark E. Stickel. Elimination of inference channels by optimal upgrading. In Proceedings of the 1994 IEEE Symposium on Research in Security and Privacy, pages 168-174, May 1994.

[12] Bhavani Thuraisingham. The use of conceptual structures for handling the inference problem. In Proc. 5th IFIP WG11.3 Workshop on Database Security, pages 333-362, November 1991.

[13] Raymond Yip and Karl Levitt. Data level inference detection in database systems. In Proc. 11th IEEE Computer Security Foundations Workshop, pages 179-189, June 1998. 


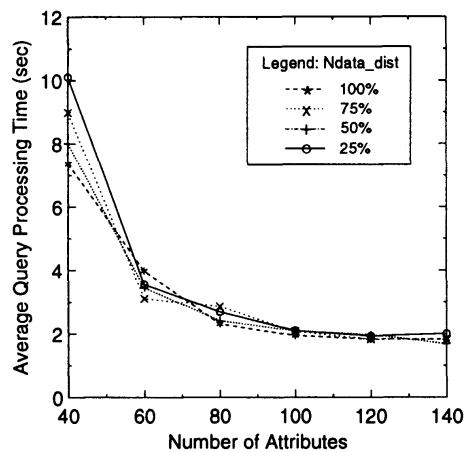

Figure 16.2 Effect of the Number of At- Figure 16.5 Effect of the Size of the Setributes in the Database.

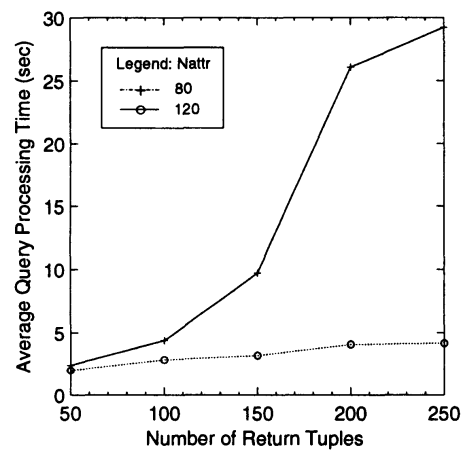

Figure 16.3 Effect of the Number of Return Tuples of Queries.

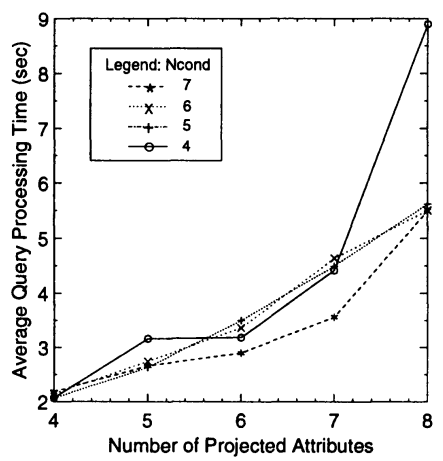

Figure 16.4 Effect of the Number of Projected Attributes of Queries.

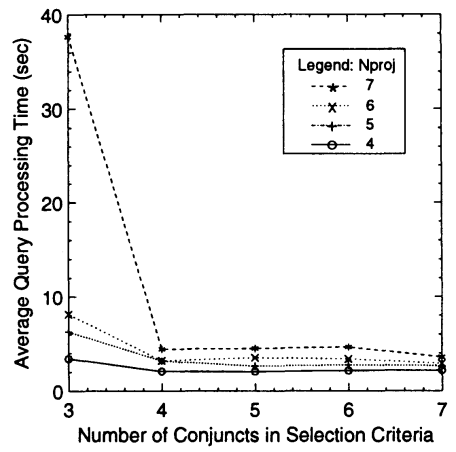

lection Criteria of Queries.

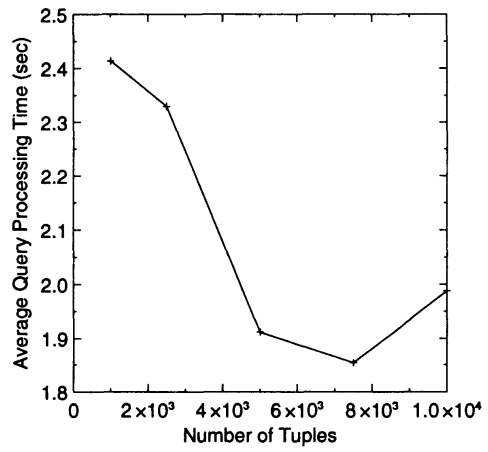

Figure 16.6 Effect of the Number of Tuples in the Database.

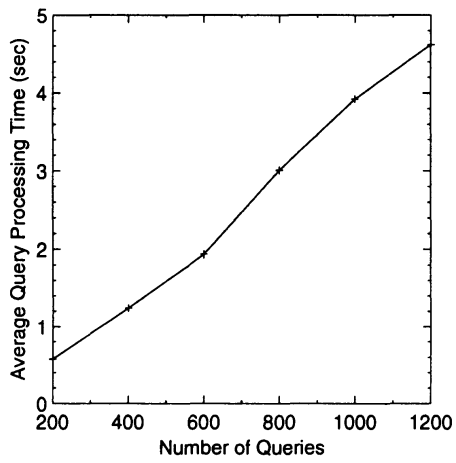

Figure 16.7 Effect of the Number of Queries Issued. 